\title{
Usefulness of Biocontrol of Pests in Tea: A Mathematical Model
}

\author{
A. Maiti ${ }^{a 1}$, A. K. Pal ${ }^{b}$ and G. P. Samanta ${ }^{c}$ \\ ${ }^{a}$ Department of Mathematics, Presidency College, Kolkata-700073, INDIA \\ ${ }^{b}$ Department of Mathematics, St. Thomas' College of Engineering and Technology \\ Kolkata-700023, INDIA \\ ${ }^{c}$ Department of Mathematics, Bengal Engineering and Science University, Shibpur \\ Howrah - 711 103, INDIA
}

\begin{abstract}
Nowadays there has been a growing consciousness among the tea industry to reduce the use of the chemical pesticides for pest control. Predators are beneficial insects that feed on harmful insects and mites, which incur considerable loss of production of tea. In this paper we have considered a tritrophic model consisting of tea plant, pest and predator to analyze different field observations. The effect of discrete time-delay on the tritrophic model is studied critically. The dynamical behaviours are studied both analytically and numerically by computer simulation. Our results suggest some theoretical measures which may be implemented for successful biological control.
\end{abstract}

Key words: natural enemy, time-delay, stability, bifurcation

AMS subject classification: 92D25

\section{Introduction}

Tea is a popular beverage made from the leaves of the evergreen shrub or tree Camellia sinensis. Known in China as early as 2737 BC, tea was first brought to Europe in 1610 and rapidly became a fashionable drink. The plant is indigenous to Assam (India) and probably to parts of China and Japan. Robert Bruce discovered the tea plant in upper Assam in 1834, which laid the foundation of tea plantation and tea industry in India. Plantations were later established in Sri Lanka and Bangladesh; producers today include Africa, South America, Georgia, Azerbaijan, Indonesia and

\footnotetext{
${ }^{1}$ Corresponding author. E-mail: alakesh_maity@ hotmail.com
} 
Iran. Under natural conditions, a tea plant grows to a small tree but it is configured into a bush by sequential pruning and other silvicultural practices, viz. tipping, plucking and by harvesting the optimum vegetative produce.

Tea production in all parts of the world is greatly hindered due to a number of pests. Various insects, mites, nematodes are major yield depressants. It is well known that approximately one third of world food production is lost due to pests. Pesticides have a great role to destroy pests and to increase crop yield. But excessive use of pesticides exerts harmful effects on human health. Most of the pesticide residues affect the central nervous system, respiratory system and gastro intestinal system of human beings. Pesticides also cause depressions, insomnia, hyper-reflexia of man. In Thailand, heavy use of chemical pesticides has taken serious turn on the 'Hmong Farmers' health. In India, more than 665 people died in the year 1889-90 because of pesticide poisoning [12]. Beside this, the resistance of pests against pesticides is increasing day by day. Pesticides also found to affect the population of Azotobacter, Rhizobium and nitrifying bacteria viz. Nitrosomonas and Nitrobacter. The list of adverse effects of chemical pesticides is quite long and this emphasized the need to search for an alternative method, which would be effective in controlling the pests, and at the same time reduce risk of health hazards. To sustain the present status of tea yield or even to improve upon today's production, use of various predators/parasitoids/pathogens (which are natural enemies of tea pests) is receiving much importance. It is seen that natural enemies of pests of tea may indirectly enhance the fitness of tea plants by destroying harmful pests. This approach of controlling harmful pests is known as biological control or simply biocontrol of pests [12]. Tea scientists have mentioned that the participation of the third trophic level members (natural enemies of pests) can provide an essential foundation for designing effective biological control of pests of tea $[5,6,12,21,23,24]$. They have earmarked some predators/parasitoids/pathogens as natural enemies of tea pests, some of which are listed in Table 1.

A chief task of theoretical ecologists is to focus on suitable non-linear models that can help us to understand the diverse array of observed scenarios in the field. In this paper we have considered a mathematical model consisting of a host (tea plant), a pest (viz. Looper Caterpiller) which destroys (or feeds on) tea plants and a predator (or parasitoid/ pathogen) (viz. Sarcophaga sp.) which is natural enemy to the concerned pest. Numerous three-species models have been proposed and studied in the ecological literature, but most of the analyses are numerical, without any analytic guide $[3,11,15,16,20]$. Not that we undervalue such works, far from it, but analytic results are important because they can show the dynamical behaviour of a class of models, rather than a particular model. This is particularly important in ecology, where confidence in exact form of any particular model is weak.

Ordinary differential equations have long played an important role in the history of theoretical ecology, and they will, no doubt, continue to serve as an indispensable tool in future investigations. However, they are generally the first approximations of the considered real system. More realistic models should include some of the past states of these systems; that is, ideally, a real system should be modelled by differential equations with time-delays. Now it is well understood that many of the processes, both natural and manmade, in biology, medicine, et cetera, involve time-delays. Timedelays occur so often, in almost every situation, that to ignore them is to ignore reality. Kuang [13] mentioned that animals must take time to digest their food before further activities and responses 
take place and hence any model of species dynamics without delays is an approximation at best. Now it is beyond doubt that in an improved analysis, the effect of time-delay due to the time required in going from egg stage to the adult stage, gestation period, et cetera, has to be taken into account. Detailed arguments on importance and usefulness of time-delays in realistic models may be found in the classical books of Gopalsamy [10], Kuang [13] and Macdonald [17].

\begin{tabular}{|c|c|c|}
\hline Pest & $\begin{array}{c}\text { Main part of } \\
\text { tea plant attacked }\end{array}$ & Natural Enemies \\
\hline $\begin{array}{l}\text { Red Spider Mite } \\
\text { (Oligonychus coffeae) }\end{array}$ & $\begin{array}{l}\text { Upper surface of } \\
\text { mature leaves }\end{array}$ & $\begin{array}{r}\text { Stethorus gilvifrons sp. } \\
\text { Chrysoperia camea }\end{array}$ \\
\hline $\begin{array}{l}\text { Tea Mosquito Bug } \\
\text { (Helopeltis theivora) }\end{array}$ & $\begin{array}{l}\text { Young leaves, } \\
\text { shoots and buds }\end{array}$ & $\begin{array}{l}\text { Melamphus sp. } \\
\text { Hexanemus sp. }\end{array}$ \\
\hline $\begin{array}{l}\text { Pink Mite } \\
\text { (Acaphylia theae) }\end{array}$ & $\begin{array}{l}\text { Both surface of } \\
\text { mature leaves }\end{array}$ & Amblyseius herbicolus \\
\hline $\begin{array}{l}\text { Kanzawa spider mite } \\
\text { (Tetranychus kanzawai) }\end{array}$ & Leaves & $\begin{array}{l}\text { Amblyseius womersleyi } \\
\text { Phytoseiulus persimilis }\end{array}$ \\
\hline Looper Caterpillar & $\begin{array}{l}\text { Young and } \\
\text { mature leaves }\end{array}$ & $\begin{array}{l}\text { Sarcophaga sp. } \\
\text { Sarcophaga sp. }\end{array}$ \\
\hline $\begin{array}{l}\text { Green fly } \\
\text { (Empoasca flavescens) }\end{array}$ & $\begin{array}{l}\text { Young leaves } \\
\text { and shoots }\end{array}$ & Dyrid cephalosporium \\
\hline
\end{tabular}

Table 1: Natural enemies of some harmful tea pests

It is a fact that mathematicians have made a considerable impact to understand several biological phenomena, but no attempt has been made for giving a strong theoretical support to the approach of biocontrol of tea pests. We have theoretically established the potential of the method of biocontrol to manage or eradicate tea pests. Further, proper suggestion of mechanisms for successful biocontrol is largely lacking. Our endeavour may accomplish such a necessity also.

The present paper deals with the qualitative study of the bizarre behaviour of the three-species 'tea plant-pest-predator' model without and with time delay. We have discussed how our mathematical findings reconcile with field observations. We have derived a criterion for complete eradication of pests and also suggested a plausible control mechanism.

The rest of the paper is structured as follows. In section 2, the basic mathematical model is introduced. A blend of detailed analysis of the dynamical behaviours of the model including boundedness, persistence and stability is presented in section 3 . In section 4 , the basic model is modified involving discrete time-delay in it and stability of the time-delayed system is discussed. 
Numerical verification of our analytical results is incorporated in Section 5 through computer simulation. Section 6 contains the general discussions of the paper and biological implications of our mathematical findings.

\section{The basic model}

Before we introduce the basic model, we would like to present a brief sketch of the construction of the model, which will indicate the biological relevance of the model.

1. We have three populations namely the host (tea plant) whose population density at time $t$ is denoted by $X$, the pest whose population density is denoted by $Y$ and the predator whose population density is denoted by $Z$.

2. Behaviour of the entire community is assumed to arise from the coupling of these interacting species where $Z$, being a natural enemy of the concerned pest, prey on $Y$ and only on $Y$ and $Y$ prey on $X$ (see Figure 1). A distinctive feature of such a community is the so called domino effect: if one species dies out, all the species at higher trophic level die out as well.

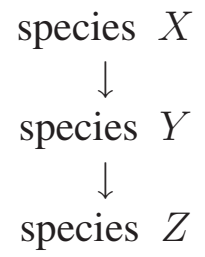

Figure 1: The feeding relationship in the food chain.

3. We assume that in the absence of the predators the prey population density grows according to a logistic curve with carrying capacity $K(K>0)$ and with an intrinsic growth rate constant $r(r>0)$. Almost every study of species dynamics undoubtedly accepted the potential of this assumption.

4. We assume that the host-pest interaction is governed by Holling type response function. Holling type response functions are very important in theoretical ecology in their own right. The most important and useful response function is the Michaelis-Menten or Holling type-II function of the form $\frac{b x}{a+x}$ where $b>0$ is the maximal growth rate of the pest and $a>0$ is the half saturation constant. Two species systems with such response function have been studied extensively and the dynamical behaviours of such systems are now very well understood [7, 19, 22]. Usually, as the degree of severity of pest attack increases, consciousness among tea industry, taking some common protective measures, and many other mechanisms often come into the picture which have a saturation effect. Therefore, Holling type-II response is supposed to be best suited for tea plantpest interaction. On the other hand, predators are being used only to destroy pests. Therefore, a Lotka-Volterra response is chosen for pest-predator interaction.

The above considerations motivate us to introduce a tritrophic food chain model under the framework of the following set of nonlinear ordinary differential equations: 


$$
\begin{array}{lll}
\frac{d X}{d t}=r X\left(1-\frac{X}{K}\right)-\frac{b X Y}{a+X}=X F(X, Y), & X(0)>0 \\
\frac{d Y}{d t}=-d Y+\frac{m X Y}{a+X}-p Y Z=Y G(X, Z), & & Y(0)>0 \\
\frac{d Z}{d t}=-\mu Z+q Y Z=Z H(Y), & Z(0)>0 .
\end{array}
$$

where $d$ denotes the death rate of the pests, $m$ is the conversion rate of the pests, $p$ is the quantity that represents the decrease in the growth rate of the pests due to predator attack, $q$ is the rate of increase of predator population and $\mu$ denotes the intrinsic mortality rate of the predators. Here all the parameters $r, K, b, a, d, m, m, q$ are positive.

\section{Dynamical behaviour of the system (2.1)}

\subsection{Boundedness}

Theorem 1. All solutions of the system (2.1) that start in $\mathbb{R}_{+}^{3}$ are uniformly bounded.

The proof of the theorem is deferred to the appendix.

\subsection{Boundary equilibria and their stability}

Computations of the boundary equilibria and their stability provide the information needed to determine the persistence of the system (2.1). In the following lemma we have mentioned the boundary equilibria of the system (2.1) and the condition of existence of them.

Lemma 2. System (2.1) always have two boundary equilibrium points $E_{0}(0,0,0)$ and $E_{1}(K, 0,0)$. The third boundary equilibrium point $E_{2}(\hat{X}, \hat{Y}, 0)$ exists if and only if $K(m-d)>a d$. When this condition is satisfied, $\hat{X}, \hat{Y}$ are given by

$$
\hat{X}=\frac{a d}{m-d}, \quad \hat{Y}=\frac{\operatorname{ram}\{K(m-d)-a d\}}{K b(m-d)^{2}} .
$$

The simplest equilibrium point is $E_{0}(0,0,0)$. The variational matrix $V\left(E_{0}\right)$ at $E_{0}$ is given by

$$
V\left(E_{0}\right)=\left[\begin{array}{ccc}
r & 0 & 0 \\
0 & -d & 0 \\
0 & 0 & -\mu
\end{array}\right]
$$

for which two of the eigenvalues are negative and one is positive, giving a point at the origin with non-empty stable manifolds and an unstable manifold. 
The equilibrium point $E_{1}(K, 0,0)$ has the variational matrix

$$
V\left(E_{1}\right)=\left[\begin{array}{ccc}
-r & \frac{b K}{a+K} & 0 \\
0 & \frac{m K}{a+K}-d & 0 \\
0 & 0 & -\mu
\end{array}\right]
$$

which has two negative and one positive eigenvalues whenever $K(m-d)>a d$ and again has non-empty stable and unstable manifolds.

The variational matrix $V\left(E_{2}\right)$ at the equilibrium point $E_{2}(\hat{X}, \hat{Y}, 0)$ is given by

$$
V\left(E_{2}\right)=\left[\begin{array}{ccc}
-\frac{r \hat{X}}{K}+\frac{b \hat{X} \hat{Y}}{(a+\hat{X})^{2}} & -\frac{b \hat{X}}{a+\hat{X}} & 0 \\
\frac{m a \hat{Y}}{(a+\hat{X})^{2}} & 0 & -p \hat{Y} \\
0 & 0 & -\mu+q \hat{Y}
\end{array}\right]
$$

The characteristic equation of $V\left(E_{2}\right)$ is

$$
\left(\lambda^{2}+B \lambda+C\right)(\lambda+\mu-q \hat{Y})=0,
$$

where $B=\frac{r \hat{X}\{a(m+d)-K(m-d)\}}{K a m}$ and $C=\frac{a b m \hat{X} \hat{Y}}{(a+\hat{X})^{3}}>0$.

The eigenvalues of $V\left(E_{2}\right)$ are

$$
\lambda_{1,2}=-B \pm \sqrt{B^{2}-4 C}, \quad \lambda_{3}=-\mu+q \hat{Y} .
$$

If $B>0$, then $\lambda_{1}$ and $\lambda_{2}$ are negative (since $C>0$ ). This implies that if $a d<K(m-d)<$ $a(m+d)$ then $E_{2}$ is locally asymptotically stable in $X Y$-plane. It can be shown that $E_{2}$ is globally asymptotically stable in $X Y$-plane whenever it is locally asymptotically stable there (see [19]).

Now $E_{2}$ is asymptotically stable in $Z$-direction if and only if the following two conditions are satisfied:

$$
\text { (i) } a d<K(m-d)<a(m+d) \text { and }(i i)-\mu+q \hat{Y}<0 .
$$

\subsection{Persistence}

From biological point of view, persistence of a system means the survival of all populations of the system in future time. Mathematically, persistence of a system means that strictly positive solutions do not have omega limit points on the boundary of the non-negative cone. Now we state a result guaranteeing the uniform persistence of the system (2.1). 
Theorem 3. Let ad $<K(m-d)<a(m+d)$. Further assume that there exists a finite number (say, $n)$ of periodic solutions $X=\phi_{i}(t), \quad Y=\psi_{i}(t), i=1,2, ., n$ in the $X Y$-plane. Then the system (2.1) is uniformly persistent, provided for each periodic solution of period $T$,

$$
\eta_{i}=-\mu+\frac{1}{T} \int_{0}^{T} q \psi_{i}(t) d t>0, i=1,2, ., n .
$$

The proof of the theorem is given in the Appendix.

Corollary 4. Let $a d<K(m-d)<a(m+d)$ and $-\mu+q \hat{Y}>0$. If there is no limit cycle in the $X Y$-plane, system (2.1) is uniformly persistent.

Proof is obvious.

\subsection{The interior equilibrium point: existence and stability}

Lemma 5. The interior equilibrium point $E^{*}\left(X^{*}, Y^{*}, Z^{*}\right)$ of the system (2.1) exists if the following two conditions are satisfied:

$$
\begin{gathered}
(i) b \mu<a r q \text { and } \\
\text { where } R=\sqrt{(K-a)^{2}-4 K\left(\frac{b \mu}{r q}-a\right)}(R+K)(m-d)>a(m+d) \\
\quad X^{*}=\frac{1}{2}(R+K-a), \quad Y^{*}=\frac{\mu}{q} \text { and } Z^{*}=\frac{(R+K)(m-d)-a(m+d)}{p(R+K+a)} .
\end{gathered}
$$

Theorem 6. If $E^{*}\left(X^{*}, Y^{*}, Z^{*}\right)$ exists, then it is locally asymptotically stable.

The proof of the theorem is given in the Appendix.

Theorem 7. If $E^{*}\left(X^{*}, Y^{*}, Z^{*}\right)$ is locally asymptotically stable, then it is globally asymptotically stable.

The proof of the theorem is deferred to the Appendix.

\section{Model with discrete delay}

It is already mentioned that time-delay is an important factor in biological systems. As a starting point of this section, we consider the following generalization of the model (2.1) involving discrete 
delay :

$$
\begin{array}{ll}
\frac{d X}{d t}=r X\left(1-\frac{X}{K}\right)-\frac{b X Y}{a+X}, & X(0)>0 \\
\frac{d Y}{d t}=-d Y+\frac{m X Y}{a+X}-p Y Z, & Y(0)>0 \\
\frac{d Z}{d t}=-\mu Z+q Y(t-\tau) Z, & Z(0)>0 .
\end{array}
$$

The delay $\tau$ in (4.1) can be regarded as a gestation period or reaction time of the predator $Z$. The delay of this form (which accounts for gestation period or reaction time of the predator) has been suggested by Beretta and Kuang [1], Freedman and Rao [8], Kuang [13], Maiti et al. [18], and many others. The system (4.1) has the same equilibria as in the previous case.

Theorem 8. Assume that the system (4.1) is point dissipative. Then the system (2.1) and (4.1) behave identically with respect to persistence.

The theorem immediately follows from the proof of Theorem 3, as the variational matrices are same in both the cases.

The main purpose of this section is to study the stability behaviour of $E^{*}\left(X^{*}, Y^{*}, Z^{*}\right)$ in presence of discrete delay $\tau \neq 0$. We linearize the system (4.1) by using the following transformations:

$$
X=X^{*}+u, \quad Y=Y^{*}+v, \quad Z=Z^{*}+w .
$$

Then the linear system is given by

$$
\frac{d U}{d t}=A U(t)+B U(t-\tau)
$$

where

and

$$
U=[u, v, w]^{T}, \quad A=\left(a_{i j}\right)_{3 \times 3}, \quad B=\left(b_{i j}\right)_{3 \times 3},
$$

$$
a_{11}=-\frac{r X^{*}}{K}+\frac{b X^{*} Y^{*}}{\left(a+X^{*}\right)^{2}}, \quad a_{12}=-\frac{b X^{*}}{a+X^{*}}, \quad a_{21}=\frac{m a Y^{*}}{\left(a+X^{*}\right)^{2}}, \quad a_{23}=p Y^{*}, \quad b_{23}=q Z^{*},
$$

and all other $a_{i j}=b_{i j}=0$.

We look for solution of the model (4.2) of the form $U(t)=\rho e^{\lambda t}, \quad 0 \neq \rho \in \mathbb{R}^{3}$. This leads to the following characteristic equation:

$$
\lambda^{3}-a_{1} \lambda^{2}+a_{2} \lambda+a_{3}\left(a_{1}+\lambda\right) e^{-\lambda \tau}=0
$$

where $a_{1}=-a_{11}, a_{2}=-a_{12} a_{21}$ and $a_{3}=a_{23} b_{32}$.

It is well known that the signs of the real parts of the solutions of (4.3) characterize the stability behaviour of $E^{*}$. Therefore, substituting $\lambda=\xi+i \eta$ in (4.3) we obtain real and imaginary parts, respectively, as

$$
\xi^{3}-3 \xi \eta^{2}+a_{1}\left(\xi^{2}-\eta^{2}\right)+a_{2} \xi+a_{3}\left\{\left(a_{1}+\xi\right) \cos \eta \tau+\eta \sin \eta \tau\right\} e^{-\xi \tau}=0,
$$


and

$$
\eta\left(3 \xi^{2}-\eta^{2}\right)+2 a_{1} \xi \eta+a_{2} \eta+a_{3}\left\{\eta \cos \eta \tau-\left(a_{1}+\xi\right) \sin \eta \tau\right\} \tau e^{-\xi \tau}=0 .
$$

A necessary condition for a stability change of $E^{*}$ is that the characteristic equation (4.3) has purely imaginary solutions. Hence to obtain the stability criterion, we set $\xi=0$ in (4.4) and (4.5) to obtain

$$
a_{1} \eta^{2}=a_{3}\left(a_{1} \cos \eta \tau+\eta \sin \eta \tau\right)
$$

and

$$
-\eta^{3}+a_{2} \eta=-a_{3}\left(\eta \cos \eta \tau-a_{1} \sin \eta \tau\right) .
$$

Eliminating $\tau$ by squaring and adding (4.6) and (4.7), we get the equation for determining $\eta$ as

$$
\eta^{6}+d_{1} \eta^{4}+d_{2} \eta^{2}+d_{3}=0
$$

where $\quad d_{1}=a_{1}^{2}-2 a_{2}, \quad d_{2}=a_{2}^{2}-a_{3}^{2}, \quad d_{3}=-a_{1}^{2} a_{3}^{2}$.

Substituting $\eta^{2}=\sigma$ in (4.8), we get a cubic equation given by

$$
F(\sigma) \equiv \sigma^{3}+d_{1} \sigma^{2}+d_{2} \sigma+d_{3}=0 .
$$

We notice that $F$ is continuous everywhere with $F(0)<0$ and $\lim _{\sigma \rightarrow \infty} F(\sigma)=\infty$. Therefore, the cubic (4.9) always has at least one positive root. Consequently the stability criteria of the system for $\tau=0$ (given in Theorem 6) will not necessarily ensure the stability of the system for $\tau \neq 0$. In the following theorem, we have given a criterion for switching the stability behaviour of $E^{*}$. The proof of the theorem is deferred to the Appendix.

Theorem 9. Let $E^{*}$ exists and $\sigma_{0}=\eta_{0}^{2}$ be a positive root of (4.9). Then there exists a $\tau=\tau^{*}$ such that $E^{*}$ is locally asymptotically stable for $0 \leq \tau<\tau^{*}$ and unstable for $\tau>\tau^{*}$, provided

$$
\begin{gathered}
f\left(\eta_{0}\right)=\left(a_{2}-3 \eta_{0}^{2}\right)\left(a_{2}-\eta_{0}^{2}\right)+\left(2 a_{1}^{2} \eta_{0}^{2}-a_{3}^{2}\right)>0, \\
\tau^{*}=\min g\left(\eta_{0}\right),
\end{gathered}
$$

where $g\left(\eta_{0}\right)=\frac{1}{\eta_{0}} \arcsin \left[\left(a_{1} a_{2} \eta_{0}\right) /\left\{a_{3}\left(\eta_{0}^{2}+a_{1}^{2}\right)\right\}\right]$ and the minimum is taken over all positive $\eta_{0}$ such that $\eta_{0}^{2}$ is a solution of the equation (4.9).

Or, in other words, the system (4.1) exhibits a Hopf-bifurcation near $E^{*}$ for $\tau=\tau^{*}$.

\section{Numerical simulations}

In this section we present computer simulation of some solutions of the system (2.1) and (4.1) to illustrate our analytical findings. We choose the parameters of the system (2.1) as $r=1, K=$ $2, a=1, b=1, d=1, m=3, p=1, \mu=1, q=1.5$ and $(X(0), Y(0), Z(0))=(2,2,2)$. Then the conditions of Theorem 6 and 7 are satisfied and $E^{*}\left(X^{*}, Y^{*}, Z^{*}\right)=(1.4574,0.6667,0.7792)$ is asymptotically stable. The phase portrait is shown in Figure 2(a). Clearly the solution is a stable spiral converging to $E^{*}$. Figure 2(b) shows that $X, Y$ and $Z$ populations approach to their steady-state values $X^{*}, Y^{*}$ and $Z^{*}$ respectively as time progresses. 


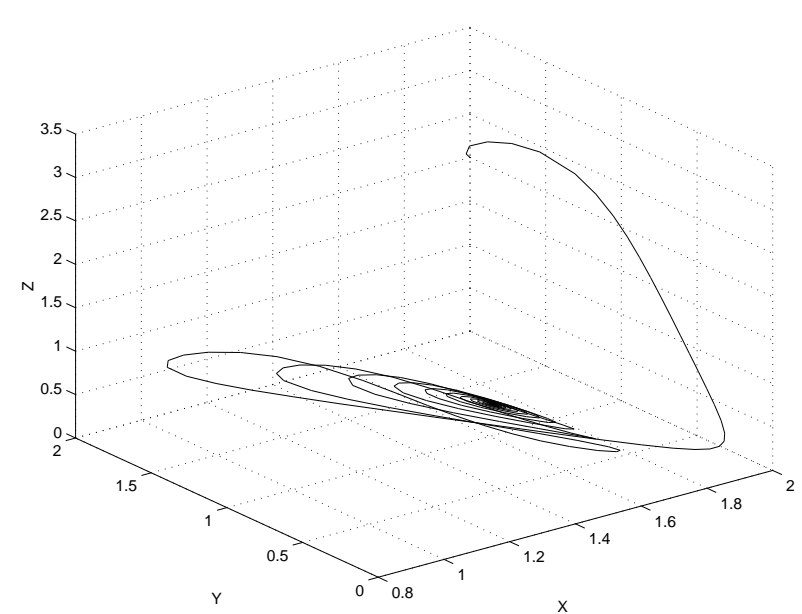

(a)

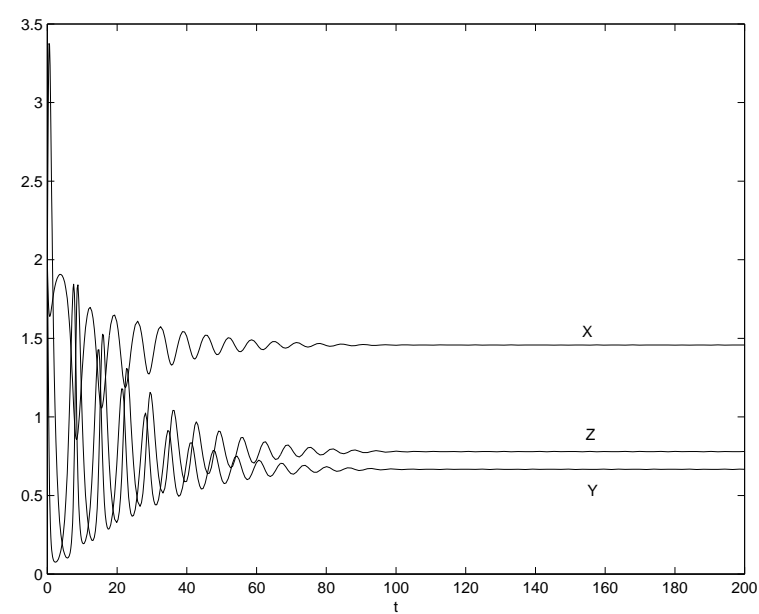

(b)

Figure 2: Here $X(0)=2, Y(0)=2, Z(0)=2$ and $r=1, K=2, a=1, b=1, d=$ $1, m=3, p=1, \mu=1, q=1.5$. (a) Phase portrait of the system (2.1) showing that $E^{*}$ is locally asymptotically stable. (b) Behaviour of the three populations with respect to time (the top curve depicts $X(t)$, the middle one depicts $Z(t)$ and the bottom one depicts $Y(t)$ ).

It is already mentioned that the stability criteria in the absence of delay ( $\tau=0$ ) will not necessarily guarantee the stability of the system in presence of delay $(\tau \neq 0)$. For the above choice of parameters, from Theorem 9, we see that there is a unique positive root of (4.9) given by $\sigma_{0}=$ $\eta_{0}^{2}=0.9199$ for which $f\left(\eta_{0}\right)=1.8405>0$ and Hopf-bifurcation occurs at $\tau=\tau^{*}=0.1154$. We see that for $\tau=0.09<\tau^{*}, E^{*}\left(X^{*}, Y^{*}, Z^{*}\right)=(1.4574,0.6667,0.7792)$ is locally asymptotically stable. The $X Y$-plane and $Y Z$-plane projections of the solution are presented in Figure 3(a) and Figure 3(b), respectively.

If we gradually increase the value of $\tau$ (keeping other parameters fixed), it is seen that $E^{*}$ loses its stability at $\tau=\tau^{*}=0.1154$. For $\tau=0.13>\tau^{*}, E^{*}$ is unstable and there is a bifurcating periodic solution near $E^{*}$ (see Figure 4(a)). The oscillations of $X, Y, Z$ populations in time are shown in Figure 4(b).

\section{Conclusions}

Time when entire globe is concerned about the menace of hazardous pesticides and means to find alternatives to chemical pesticides, tea industry cannot remain apathetic. Tea is consumed directly through infusion in water, without being washed with water. Hence, industry should endeavour to use minimum possible chemical pesticides to avoid pollution and health problems. Various predators, parasitoids and pathogens are found to be effective against different tea pests. This non-conventional method of pest control by using beneficial predators or parasitoids, which feed on harmful pests and thus help to keep the pest populations down, is getting importance day by 


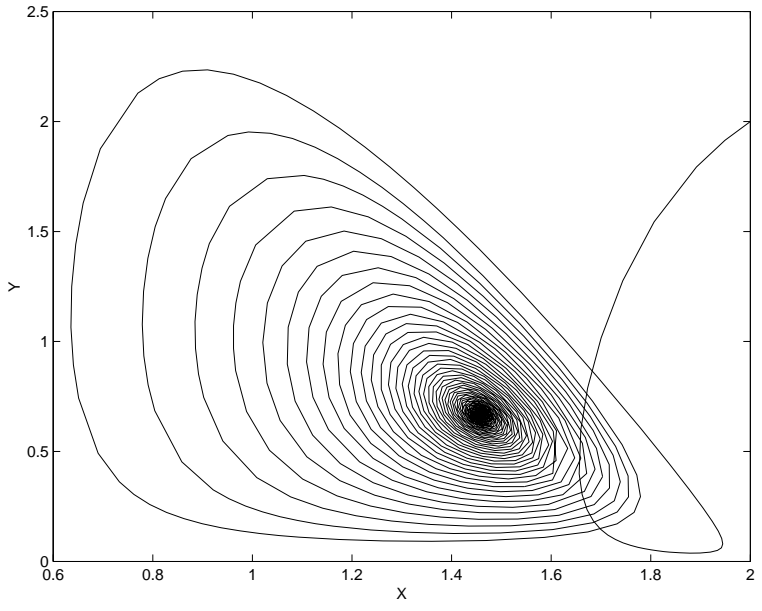

(a)

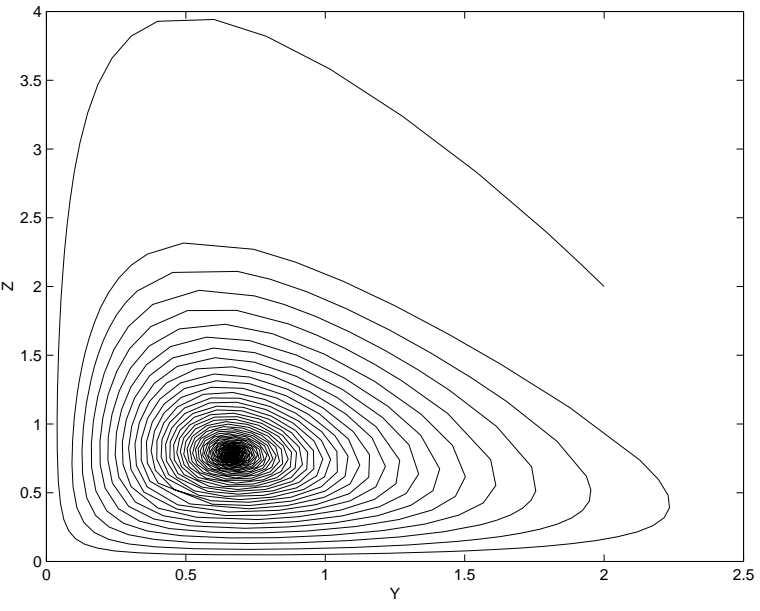

(b)

Figure 3: Here $X(0)=2, Y(0)=2, Z(0)=2$ and $r=1, K=2, a=1, b=1, d=1, m=$ $3, p=1, \mu=1, q=1.5$ and $\tau=0.09<\tau^{*}$. (a) $X Y$-plane projection of the solution. (b) $Y Z$-plane projection of the solution.

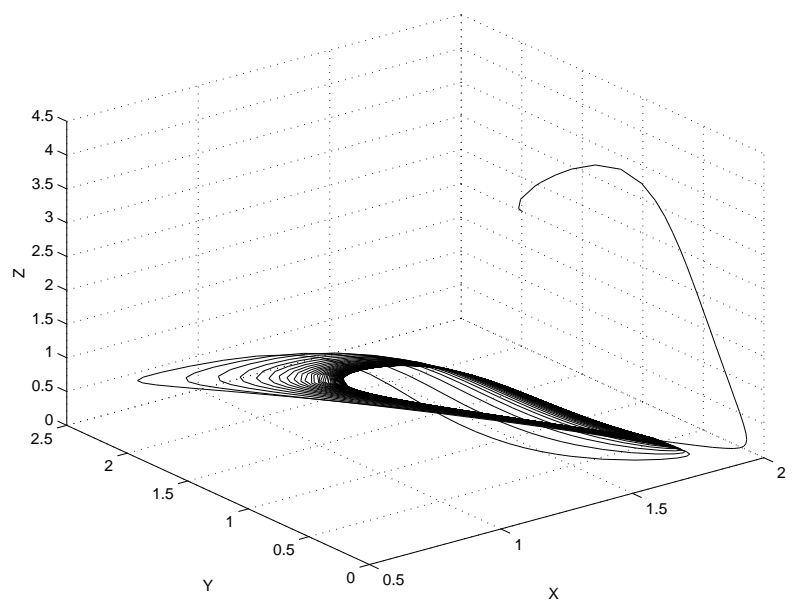

(a)

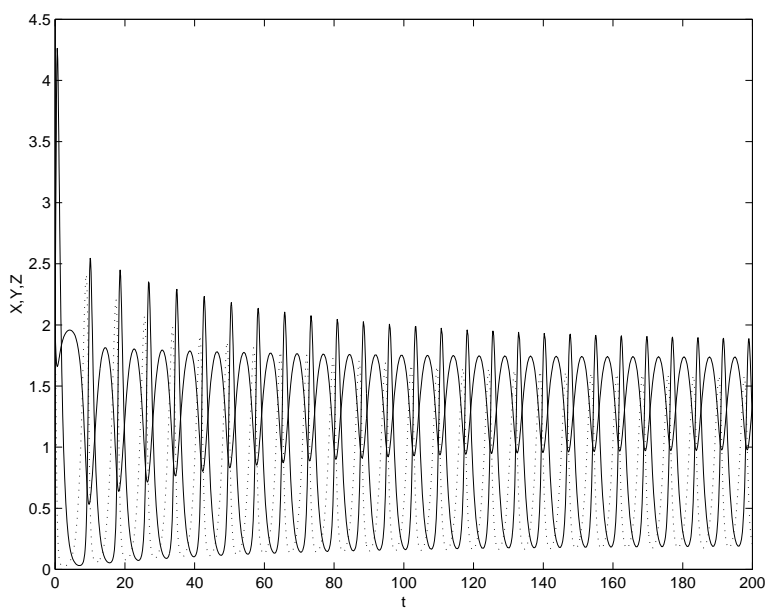

(b)

Figure 4: Here all other parameter values are same as in Figure 3 except $\tau=0.13>\tau^{*}$. (a) Phase portrait of the system (4.1) showing a limit cycle which grows out of $E^{*}$. (b) Oscillation of $X, Y, Z$ populations respectively in time. 
day in its own right. In an experiment conducted by Das and Barua [5], it is found that Bacillus thuringiensis is very effective against looper caterpillar and hence it may determine the fitness of caterpillar affected tea plants. Recently many such experiments have been performed by several researchers on different pests to prove the potential of this technique in controlling major tea pests $[21,23,24]$.

In this paper we have studied the usefulness of this approach with the help of mathematical modelling. The basic model (2.1) is considered by analyzing the theoretical and experimental findings of several researchers. The response function for tea plant and pest is taken of Holling type-II, whereas the interaction between pest and predator is assumed to be governed by the LotkaVolterra response function. Our theoretical study of dynamical behaviours of the system (2.1) is started by showing the uniform boundedness of the system which, in turn, implies that the system (2.1) is biologically well behaved. Also boundedness of a system together with the stability analysis of the boundary equilibria provide all the necessary information to establish persistence of the system. Theorem 3 and Corollary 4 give the criteria for uniform persistence of the system when there is a finite number of periodic solutions and no periodic solution, respectively. The persistence criteria should be well implemented for long time survival of the populations of the system.

Our study is capable of providing a condition for complete eradication of pests. This condition is $a>K(m-d) / d$. This indicates that if the degree of consciousness is very high and different protective measures are taken very seriously, then the pest may be eradicated. But it is a fact that degree of consciousness always vary, and there is many other factors also. Perhaps for this reason, in case of a severe attack of pests, complete eradication of pests cannot be done successfully, except in some very rare occasions. That is why, the mechanism for pest control may be very useful and practically more desirable. This indicates the usefulness of the criterion for coexistence of the three species. Here it is observed that the three-species coexisting equilibrium exists if $b \mu<$ arq and $(R+K)(m-d)>a(m+d)$. Then Theorem 6 and Theorem 7 respectively show that if $E^{*}$ exists, then it is locally and globally asymptotically stable.

Now we make an important observation that the size of the tea plant population $(X)$ in the absence and presence of beneficial predator $(Z)$ are $\hat{X}$ and $X^{*}$ respectively so that $X^{*}-\hat{X}=$ $\{(R+K)(m-d)-a(m+d)\} /\{2(m-d)\}>0$. This guarantees that predator attack of pests enhance fitness of tea plant. At the same time, since $E^{*}$ is globally asymptotically stable whenever it exists, we find that $Y^{*}-\hat{Y}=-\operatorname{arq} d\{K(m-d)-a m\} /\left\{K b q(m-d)^{2}\right\}<0$, where $\hat{Y}$ and $Y^{*}$ are respectively the size of the pest population in absence and presence of predators. This indicates that the pest population plunges into depression by the attack of predators. This result is not only in good agreement with experimental observations of Das and Barua [5], Mochizuki [21], Takafuji et al. [23], Takahashi et al. [24], and of many other researchers, but it gives a strong theoretical support to such a non-conventional approach of pest control of tea.

It is mentioned by several researchers that the effect of time delay must be taken into account in order to have a biologically useful mathematical model $[10,13,17]$. From this viewpoint, we have formulated the model (4.1) where the delay may be looked upon as the gestation period or reaction time of the predator $(Z)$. Then a rigorous analysis lead us to the Theorem 9 which mention that the stability criteria in absence of delay is no longer enough to guarantee the stability in presence of 
delay, rather there is a value $\tau^{*}$ of the delay $\tau$ such that the system is stable for $\tau<\tau^{*}$ and become unstable for $\tau>\tau^{*}$.

All our important mathematical findings with and without time-delay are numerically verified in section 5 and graphical representation of a variety of solutions of the systems (2.1) and (4.1) are depicted using MATLAB. The numerical study presented here also shows that, if the time-delay is small enough, then the population levels may be kept at a required level, but if it the time-delay lies above the critical value, then the system is driven to an unstable state.

The conditions for stable coexistence of the tea plant, pest and predator can be written as $b>$ arq/mu and $m>d(R+K+a) /(R+K-a)$. This implies that if the pest is aggressive (this may be characterized by large $b$ and $m$ ), then an aggressive predator (characterized by small gestation period) can only manage the pest. Therefore it is very important to select the suitable predator (natural enemy) corresponding to the concerned pest, especially when the pest is aggressive. The field experiments mentioned above are in good agreement with this result.

\section{Acknowledgements}

We are grateful to the referees for their careful reading and constructive comments. We also wish to thank Dr. Sergei Petrovskii, Associate editor, MMNP, for his valuable suggestions.

\section{Appendix}

Proof of Theorem 1. Let $(X(t), Y(t), Z(t))$ be any solution of the system (2.1) with positive initial conditions.

Since

$$
\frac{d X}{d t} \leq r X\left(1-\frac{X}{K}\right)
$$

by a standard comparison theorem, we have

$$
\limsup _{t \rightarrow \infty} X(t) \leq K
$$

Let

$$
W=\frac{m}{b} X+Y+\frac{p}{q} Z
$$

Then

$$
\begin{aligned}
\frac{d W}{d t} & =\frac{r m}{b} X\left(1-\frac{X}{K}\right)-d Y-\frac{p \mu}{q} Z \\
& \leq \frac{2 r m}{b} X-\kappa W, \text { where } \kappa=\min \{r, d, \mu\}
\end{aligned}
$$

Therefore

$$
\frac{d W}{d t}+\kappa W \leq \frac{2 r m}{b}
$$


Applying a theorem on differential inequalities [2], we obtain

$$
0 \leq W(X, Y, Z) \leq \frac{2 r m}{b \kappa}+\frac{W(X(0), Y(0), Z(0))}{e^{\kappa t}}
$$

and for $t \rightarrow \infty$,

$$
0 \leq W \leq \frac{2 r m}{b \kappa}
$$

Thus, when $\tau=0$, all the solutions of the system (1.2) enter into the region

$$
B=\left\{(X, Y, Z): 0 \leq W \leq \frac{2 r m}{b \kappa}+\epsilon, \text { for any } \epsilon>0\right\}
$$

This proves the theorem.

Proof of Theorem 3. Let $\theta(x)$ be the orbit through the point $x=(X, Y, Z)$ and $\Omega(x)$ be the omega-limit set of the orbit through $x$. Note that $\Omega(x)$ is bounded.

We claim that $E_{0}$ does not belong to $\Omega(x)$. If $E_{0} \in \Omega(x)$, by Butler-McGehee lemma [9], there exists a point $P$ in $\Omega(x) \cap W^{S}\left(E_{0}\right)$ where $W^{S}\left(E_{0}\right)$ denotes the stable manifold of $E_{0}$. Since $\theta(P)$ lies in $\Omega(x)$ and $W^{S}\left(E_{0}\right)$ is the $Y Z$-plane, we conclude that $\theta(P)$ is unbounded, which is a contradiction.

Next $E_{1}$ does not belong to $\Omega(x)$, for otherwise, since $E_{1}$ is a saddle point, which follows from the condition $K(m-d)>a d$, by Butler McGehee lemma, there exists a point $P$ in $\Omega(x) \cap W^{S}\left(E_{1}\right)$. Now $W^{S}\left(E_{1}\right)$ is the $X$-axis implies that an unbounded orbit lies in $\Omega(x)$, which is contrary to the boundedness of the system.

Lastly we show that no periodic orbit in the $X Y$-plane or $E_{2}$ belongs to $\Omega(x)$. The condition $a d<K(m-d)<a(m+d)$ implies that the eigenvalues $\lambda_{1}$ and $\lambda_{2}$ of $V\left(E_{2}\right)$ have negative real parts. Let $\gamma_{i}(i=1,2, n)$ denotes the closed orbit of the periodic solution $\left(\phi_{i}(t), \psi_{i}(t)\right)$ in $X Y$-plane such that $\gamma_{i}$ lies inside $\gamma_{i-1}$. The variational matrix $V_{i}\left(\phi_{i}(t), \psi_{i}(t), 0\right)$ corresponding to $\gamma_{i}$ is given by

$$
V_{i}=\left[\begin{array}{ccc}
F\left(\phi_{i}(t), \psi_{i}(t)\right)+F_{X}\left(\phi_{i}(t), \psi_{i}(t)\right) & \phi_{i}(t) F_{Y}\left(\phi_{i}(t), \psi_{i}(t)\right) & 0 \\
\psi_{i}(t) G_{X}\left(\phi_{i}(t), 0\right) & G\left(\phi_{i}(t), 0\right) & \psi_{i}(t) G_{Z}\left(\phi_{i}(t), 0\right) \\
0 & 0 & H\left(\psi_{i}(t)\right)
\end{array}\right]
$$

Here $H\left(\psi_{i}(t)\right)=-\mu+q \psi_{i}(t)$. Computing the fundamental matrix of the linear periodic system

$$
M^{\prime}=V_{i}(t) M, \quad M(0)=I,
$$

we find that its Floquet multiplier in the $z$-direction is $e^{\eta_{i}(t)}$. Then proceeding in an analogous manner like Kumar and Freedman [14], we conclude that no $\gamma_{i}$ lies in $\Omega(x)$. Thus $\Omega(x)$ lies in the positive octant and system (2.1) is persistent. Finally, since only the closed orbits and the equilibria 
form the omega limit set of the solutions on the boundary of $\mathbb{R}_{+}^{3}$ and system (2.1) is dissipative, by the main theorem in [4], the system (2.1) is uniformly persistent.

Proof of Theorem 6. The variational matrix of the system (2.1) at $E^{*}$ is given by

$$
V\left(E^{*}\right)=\left[\begin{array}{ccc}
m_{11} & m_{12} & 0 \\
m_{21} & 0 & m_{23} \\
0 & m_{32} & 0
\end{array}\right] .
$$

where

$$
m_{11}=-\frac{r X^{*}}{K}+\frac{b X^{*} Y^{*}}{\left(a+X^{*}\right)^{2}}, m_{12}=-\frac{b X^{*}}{a+X^{*}}, m_{21}=\frac{m a Y^{*}}{\left(a+X^{*}\right)^{2}}, m_{23}=-p Y^{*}, m_{33}=q Z^{*} .
$$

The characteristic equation is

$$
\lambda^{3}+A_{1} \lambda^{2}+A_{2} \lambda+A_{3}=0,
$$

where $A_{1}=-m_{11}, \quad A_{2}=-m_{12} m_{21}-m_{23} m_{32}$ and $A_{3}=-\operatorname{det} V\left(E^{*}\right)=m_{11} m_{23} m_{32}$.

Now after some simplification, we obtain

$$
A_{1}=-m_{11}=\frac{r R X^{*}}{K\left(a+X^{*}\right)}>0, \quad A_{3}=\frac{r p q R X^{*} Y^{*} Z^{*}}{K\left(a+X^{*}\right)}>0
$$

and

$$
\Delta=A_{1} A_{2}-A_{3}=\frac{r a b m R X^{* 2} Y^{*}}{K\left(a+X^{*}\right)^{4}}>0 .
$$

Since $A_{1}>0, A_{3}>0$ and $\Delta>0$, therefore $E^{*}$ is locally asymptotically stable by Routh Hurwitz criterion.

Proof of Theorem 7. Let us consider the following positive definite function about $E^{*}$ :

$$
\begin{aligned}
J(X, Y, Z)= & M\left(X-X^{*}-X^{*} \ln \frac{X}{X^{*}}\right)+\left(Y-Y^{*}-Y^{*} \ln \frac{Y}{Y^{*}}\right) \\
& +N\left(Z-Z^{*}-Z^{*} \ln \frac{Z}{Z^{*}}\right) .
\end{aligned}
$$

where $M, N$ are positive constants to be specified later on.

Differentiating $J$ with respect to $t$ along the solution of (2.1), a little algebraic manipulation yields

$$
\begin{aligned}
\frac{d J}{d t}= & -M\left\{\frac{r}{K}-\frac{b Y^{*}}{(a+X)\left(a+X^{*}\right)}\right\}\left(X-X^{*}\right)^{2} \\
& +\left\{\frac{a m-M\left(a b+b X^{*}\right)}{(a+X)\left(a+X^{*}\right)}\right\}\left(X-X^{*}\right)\left(Y-Y^{*}\right) \\
& +(-p+N q)\left(Y-Y^{*}\right)\left(Z-Z^{*}\right) .
\end{aligned}
$$


We now choose $M=\left[a m /\left\{b\left(a+X^{*}\right)\right\}\right], \quad N=(p / q)$ and notice that when $E^{*}$ exists (and consequently it is locally asymptotically stable) then

$$
\frac{r}{K}-\frac{b Y^{*}}{(a+X)\left(a+X^{*}\right)}>\frac{r X}{K(a+X)}>0
$$

Therefore $(d J / d t)$ is negative definite and consequently $J$ is a Lyapunov function with respect to all solutions in the interior of the positive orthant, proving the theorem.

Proof of Theorem 9. First of all, we note that $\eta_{0}$ is a solution of (4.8). Solving (4.6) for $\cos \eta_{0} \tau$ and substituting in (4.7), we find that for $\tau=\tau_{0}$, the characteristic equation (4.3) have purely imaginary roots, $\pm i \eta_{0}$, where $\tau_{0}=g\left(\eta_{0}\right)$. Again, if $\pm \eta_{0}$ is a solution of (4.6) and (4.7), then $\eta_{0}^{2}$ is a solution of (4.8). The smallest value of such $\tau_{0}$ is the required $\tau^{*}$. The theorem will be proved if we can show that $\left[\frac{d \xi}{d \tau}\right]_{\tau=\tau^{*}}>0$. To show this, we differentiate (4.4) and (4.5) with respect to $\tau$ and then set $\xi=0$ to obtain

$$
\begin{gathered}
C(\eta) \frac{d \xi}{d \tau}+D(\eta) \frac{d \eta}{d \tau}=G(\eta), \\
-D(\eta) \frac{d \xi}{d \tau}+C(\eta) \frac{d \eta}{d \tau}=H(\eta),
\end{gathered}
$$

where

$$
\begin{aligned}
& \left.C(\eta)=a_{2}-3 \eta^{2}+a_{3}\left(1-a_{1} \tau\right) \cos \eta \tau-a_{3} \eta \tau \sin \eta \tau\right) \\
& \left.D(\eta)=-2 a_{1} \eta+a_{3}\left(1-a_{1} \tau\right) \sin \eta \tau+a_{3} \eta \tau \cos \eta \tau\right) \\
& G(\eta)=a_{3} \eta\left(a_{1} \sin \eta \tau-\eta \cos \eta \tau\right) \\
& H(\eta)=a_{3} \eta\left(\eta \sin \eta \tau+a_{1} \cos \eta \tau\right) .
\end{aligned}
$$

Solving (A.1) and (A.2) with $\tau=\tau^{*}$ and $\eta=\eta_{0}$, we get

$$
\left[\frac{d \xi}{d \eta}\right]_{\tau=\tau^{*}}=\frac{\eta_{0}^{2} f\left(\eta_{0}\right)}{C^{2}\left(\eta_{0}\right)+D^{2}\left(\eta_{0}\right)},
$$

which is positive under the condition of the theorem and thus the theorem is established.

\section{References}

[1] E. Beretta, Y. Kuang. Convergence results in a well known delayed predator-prey system. J. Math. Anal. Appl., 204 (1996), No. 3, 840-853.

[2] G. Birkhoff, G. C. Rota. Ordinary differential equations. Ginn, Boston, 1982.

[3] M. P. Boer, B. W. Kooi, S. A. L. M. Kooijman. Homoclinic and heteroclinic orbits to a cycle in a tri-trophic food chain. J. Math. Biol., 39 (1999), No. 1, 319-328. 
[4] G. Butler, H. I. Freedman, P. Waltman. Uniformly persistent systems. Proc. Am. Math. Soc., 96 (1986), No. 3, 425-430.

[5] S. C. Das, K. C. Barua. Scope of bio-control of pests and diseases in tea plantations. Proc. Int. Conf. Tea Res. : Global Perspective, Calcutta : 119-125, 1990 (11-12 Jan).

[6] S. C. Das, M. C. Borthakur, B. Gope. Need for non-conventional approach in tea pest management., Proc. 30th Tocklai Conf., 80-89, 1988.

[7] H. I. Freedman. Deterministic mathematical models in population ecology. Marcel Dekker, New Work, 1980.

[8] H. I. Freedman, V. S. H. Rao. The trade-off between mutual interference and time lags in predator prey systems. Bull. Math. Biol., 45 (1983), No. 6, 991-1004.

[9] H. I. Freedman, P. Waltman. Persistence in models of three interacting predator-prey populations. Math. Biosci., 68 (1984), No. 2, 213-231.

[10] K. Gopalsamy. Stability and oscillations in delay differential equations of population dynamics. Kluwer Academic, Dordrecht, 1992.

[11] A. Hastings, T. Powell. Chaos in a three-species food chain. Ecology, 72 (1991), No. 3, 896-903.

[12] S. E. Kabir. Non-conventional methods of pest control in tea (Camellia sinensis L.), in Recent Advances in Animal Science Research (vol. I) (edited by S. K. Ghosal and D. Ray), Orion Press International, West Bengal, 2001, 163-167.

[13] Y. Kuang. Delay differential equations with applications in population dynamics. Academic Press, New York, 1993.

[14] R. Kumar, H. I. Freedman. A mathematical model of facultative mutualism with populations interacting in a food chain. Math. Biosci., 97 (1989), No. 2, 235-261.

[15] Y. Kuznetsov, O. De Feo, S. Rinaldi. Belyakov homoclinic bifurcation in a tritrophic food chain model. SIAM J. Appl. Math., 62 (2001), No. 2, 462-487.

[16] Y. Kuznetsov, S. Rinaldi. Remarks on food chain dynamics. Math. Biosci., 134 (1996), No. $1,1-33$.

[17] N. Macdonald. Biological delay systems : linear stability theory. Cambridge Univ. Press, Cambridge, 1989.

[18] A. Maiti, M. M. Jana, G. P. Samanta. Deterministic and stochastic analysis of a ratiodependent predator-prey system with delay. Nonlinear Analysis : Modelling and Control, 12 (2007), No. 3, 383-398. 
[19] A. Maiti, G. P. Samanta, Deterministic and stochastic analysis of a prey-dependent predatorprey system. Internat. J. Math. Educ. Sci. Technol., 36 (2005), No. 1, 65-83.

[20] K. McCann, P. Yodzis. Bifurcation structure of a three-species food chain model. Theor. Pop. Biol., 48 (1995), No. 2, 93-125.

[21] M. Mochizuki. Effectiveness and pesticide susceptibility of the pyrethroid-resistant predatory mite Amblyseius womersleyi in the integrated pest management of tea pests. BioControl, 48 (2003), No. 2, 207-221.

[22] D. Xiao, S. Ruan. Global analysis in a predator-prey system with nonmonotonic functional response. SIAM J. Appl Math., 61 (2001), No. 4, 1445-1472.

[23] A. Takafuji, A. Ozawa, H. Nernoto, T. Gotoh. Spider mites of Japan : their biology and control. Experimental and Applied Acarology, 24 (2000), No. 5-6, 319-335.

[24] F. Takahashi, M. Inoue, A. Takafuji. Management of the spider-mite population in a vinylhouse vinery by releasing Phytoseiulus persimilis Athias-Henriot onto the ground cover. Japanese J. Appl. Entomol. and Zool., 42 (1998), No. 2, 71-76. 